\title{
PERK Regulates the Sensitivity of Hepatocellular Carcinoma Cells to High-LET Carbon lons via either Apoptosis or Ferroptosis
}

\author{
Xiaogang Zheng1,2,3,4, Bingtao Liu'1,2,3,4, Xiongxiong Liu 1,2,3,4, Ping Li1,2,3,4, Pengcheng Zhang ${ }^{1,2,3,4}$, Fei Ye ${ }^{1,2,3,4}$, \\ Ting Zhao ${ }^{1,2,3,4}$, Yanbei Kuang1,2,3,4, Weiqiang Chen",2,3,4, Xiaodong Jin 1,2,3,4凶, Qiang Li 1,2,3,4凶 \\ 1. Institute of Modern Physics, Chinese Academy of Sciences, Lanzhou 730000, China. \\ 2. Key Laboratory of Heavy Ion Radiation Biology and Medicine, Chinese Academy of Sciences, Lanzhou 730000, China. \\ 3. Key Laboratory of Basic Research on Heavy Ion Radiation Application in Medicine, Gansu Province, Lanzhou 730000, China. \\ 4. University of Chinese Academy of Sciences, Beijing 100049, China. \\ $\triangle$ Corresponding authors: Dr. Xiaodong Jin, Institute of Modern Physics, Chinese Academy of Sciences, 509 Nanchang Road, Lanzhou 730000, Gansu Province, \\ China. Tel: +86-931-5196129, Fax: +86-931-8272100, E-mail: jinxd@impcas.ac.cn. Dr. Qiang Li, Institute of Modern Physics, Chinese Academy of Sciences, 509 \\ Nanchang Road, Lanzhou 730000, Gansu Province, China. Tel: +86-931-5196181, Fax: +86-931-8272100, E-mail: liqiang@impcas.ac.cn.
}

(c) The author(s). This is an open access article distributed under the terms of the Creative Commons Attribution License (https://creativecommons.org/licenses/by/4.0/). See http://ivyspring.com/terms for full terms and conditions.

Received: 2021.04.14; Accepted: 2021.12.05; Published: 2022.01.01

\begin{abstract}
PERK is one of the transmembrane sensors of unfolded protein response (UPR) triggered by ER stress. In this study, we evaluated the role of PERK in the sensitivity of hepatocellular carcinoma (HCC) cells to high linear energy transfer (LET) carbon ions $(\mathrm{Cl})$. We found that $\mathrm{Cl}$ irradiation could induce ER stress in HCC cells. On the one hand, PERK promoted autophagy via regulating ATF4 expression; on the other hand, PERK regulated p53 expression, and the latter either induced autophagy through up-regulating DRAM, or directly promoting apoptosis through the mitochondrial pathway or facilitating ferroptosis via down-regulating SLC7AII (the extrinsic pathway), but independent of GPX4 (the intrinsic pathway). These factors jointly determined the sensitivity of HCC cells to high-LET Cl radiation. Inhibiting TP53 directly increased cellular radioresistance definitely. Moreover, the death of HepG2 (TP53 wild type) cells induced by high-LET Cl irradiation combined with sorafenib treatment might be caused by a mixed-type regulated cell death $(R C D)$ including both apoptosis and ferroptosis, suggesting that apoptosis and ferroptosis are synergetic cell death modes regulated by TP53, which is one of the reasons why the sensitivity of HepG2 cells is higher than that of Hep3B (TP53 null type) and PLC/PRF5 (TP53 mutated type) cells. Therefore, our work might shed light on the potential therapeutic implication of $\mathrm{Cl}$ radiotherapy combined with PERK targeted clinical drugs to implement personalized and precise treatment of HCCs.
\end{abstract}

Key words: PERK, carbon ion, ER stress, autophagy, ferroptosis

\section{Introduction}

Ionizing radiation brings about damage to biological macromolecules such as DNA and proteins, as well as organelles such as mitochondria and endoplasmic reticulum (ER) through direct and indirect effects, causing cell cycle arrest, autophagy and ER stress, and eventually leading to cell death [1]. The radiobiological characteristics make high linear energy transfer (LET) carbon ions (CI) possess great advantages in the treatment of tumors resistant to conventional radiations [2]. At present, the main treatment modalities of HCC are surgery, liver transplantation, percutaneous or arterial intervention, small molecule multi-kinase inhibitor and systemic chemotherapy [3]. Although hepatocellular carcinoma (HCC) is sensitive to ionizing radiation, conventional radiation plays a limited role in the treatment of HCC because the tolerated dose of the liver is relatively lower [4]. Carbon ions (CI) radiation has a sharp energy deposition at the end of its range, known as the Bragg peak, which can increase the dose to the 
tumor target without increasing toxicity to surrounding non-cancerous tissues and organs, thereby bring better therapeutic efficacy on early and intermediate HCCs [5-7].

Abnormality in the ER signals or quality control mechanisms triggers a stress state named as ER stress [8]. Cells alleviate ER stress through unfolded protein response (UPR), which is regulated by three transmembrane sensors, namely PKR-like ER kinase (PERK), activating transcription factor 6 (ATF6) and inositol requiring enzyme 1 (IRE1) [9, 10]. Under normal physiological conditions, they bind to immunoglobulin heavy chain binding protein (Bip) and stay inactive [11]. Once ER stress is activated, Bip dissociates from the aforementioned transmembrane sensors, and selectively binds to the unfolded or misfolded proteins through its substrate-binding domain. After dissociation, the transmembrane sensors are activated, then a series of corresponding response procedures are initiated to reconstruct ER homeostasis and maintain cell survival [12]. However, if the steady-state reconstruction mechanism fails, the signal output of UPR will switch from pro-survival to pro-apoptotic, leading to the activation of the endogenous apoptosis [13].

PERK activation mediates phosphorylation of eIF2 $\alpha$ at Ser51, reduces the translation of common proteins and maintains cell survival. PERK also selectively translates the transcription factor ATF4 and regulates the expression of genes related to amino acid biosynthesis, oxidative stress, and apoptosis. PERK can also induce the expression of genes related to radioresistance through Nrf2 phosphorylation [14, 15]. Briefly, PERK maintains ER homeostasis and enhances the adaptability to oxidative stress through the PERK-eIF2 $\alpha-A T F 4$ and PERK-Nrf2 signal cascades, and promotes cell survival through overexpression of Bcl-2 and Bcl-XL $[9,14]$.

Persistent or intense ER stress signals can promote PERK-induced cell death. One of the mechanisms is that p53 upregulates PUMA and NOXA and induces p53-dependent apoptosis [16, 17]. The activation of PERK can up-regulate CHOP transcription, inhibit the expression of Bcl-2, while promoting the expression of pro-apoptotic BIM, and improve protein synthesis and oxidative stress, and eventually lead to apoptosis [18].

Radiation-induced UPR regulates autophagyrelated genes through PERK [19]. The induction of autophagy can reduce apoptosis while inhibiting ER stress-related autophagy can promote apoptosis and inhibit tumor progression. PERK-mediated ATF4 activation can up-regulate autophagy-related genes such as LC3, BECN1, Atg3, Atg12 and Atg16L1. Although ER stress and autophagy work independently, they share many common features, including protecting cells by reducing stress and inducing cell death under extreme conditions [20, 21].

The present study was aimed at clarifying the mechanisms underlying the sensitivity of HCC cells to high-LET CI radiation, and then providing a theoretical basis for adopting personalized and precise treatment schemes for HCC based on different TP53 statuses in clinical treatment.

\section{Materials and Methods}

\section{Cells and Reagents}

Human HCC cell lines (HepG2, Hep3B and $\mathrm{PLC} / \mathrm{PRF} / 5)$ were purchased from the Type Culture Collection of the Chinese Academy of Sciences (Shanghai, China). All cell lines were attached with STR profiling and mycoplasma contamination test reports. HepG2, Hep3B and PLC/PRF/5 have TP53 wild, null and mutant types, respectively. HepG2 cells were maintained in RPMI-1640 medium (HyClone, US), Hep3B and PLC/PRF/ 5 cells were maintained in MEM medium (HyClone, US). All cell cultures were supplemented with $10 \%(\mathrm{v} / \mathrm{v})$ fetal bovine serum (FBS) (Bailing, China), $100 \mathrm{U} / \mathrm{mL}$ penicillin and 100 $\mu \mathrm{g} / \mathrm{mL}$ streptomycin, and incubated in a humidified incubator at $37^{\circ} \mathrm{C}$ with $5 \% \mathrm{CO}_{2}$.

Chloroquine (CQ) and 4-Phenylbutyric acid (4-PBA) were purchased from Sigma-Aldrich (US). Ferrostatin-1 and Sorafenib were purchased from Selleck (US). Primary antibodies p53, PUMA, NOXA, $\mathrm{xCT}$, PTGS2 and 4-Hydroxynonenal were purchased from Abcam (UK), PERK, ATF4, pBCl-2 and GPX4 were purchased from Cell Signaling Technology (US), $\beta$-Actin, Bip, GSK3-beta, DRAM, LC3, SQSTM1 / p62 and Caspase-3 were purchased from Proteintech (China). Secondary antibodies were all purchased from Proteintech (China).

\section{Transfection with siRNA}

About $2 \times 10^{5}$ cells were inoculated into a $35 \mathrm{~mm}$ culture dish overnight, and the TP53-siRNA (RIBOBIO, China) was transfected with a riboFECT ${ }^{\mathrm{TM}}$ transfection kit according to the manufacturer's operating procedures. $5 \mathrm{ul}$ siRNA was added to $120 \mathrm{ul}$ riboFECT ${ }^{\mathrm{TM}}$ Buffer and mixed gently, then added $12 \mathrm{ul}$ riboFECT ${ }^{\mathrm{TM}}$ Reagent and mixed by pipetting repeatedly, and incubated at room temperature for $15 \mathrm{~min}$ to prepare the transfection complex. 863ul of complete medium without penicillin or streptomycin was added into the transfection complex and mixed gently. The prepared mixture was added to the culture dish containing adherent cells, $1 \mathrm{ml}$ of culture medium was reserved to make the total volume of the culture solution $2 \mathrm{ml}$. After 48 hours, the medium was 
replaced and then the cells were irradiated. The target sequence of si-h-TP53 was GACTCCAGTGGTAAT CTAC.

\section{Irradiation}

CI: $3 \times 10^{5}$ cells were inoculated into a $35 \mathrm{~mm}$ culture dish, when the cell density reaches about $80 \%$, irradiations were carried out with a CI beam of 165 $\mathrm{MeV} / \mathrm{u}$ in the heavy-ion therapy terminal of the Heavy Ion Research Facility in Lanzhou (HIRFL) at the Institute of Modern Physics, Chinese Academy of Sciences (Lanzhou, China). The LET value of the CI beams was adjusted to $50 \mathrm{keV} / \mu \mathrm{m}$ on the cell samples.

X-rays: $3 \times 10^{5}$ cells were inoculated into a $35 \mathrm{~mm}$ culture dish, when the cell density reaches about $80 \%$, irradiations were performed with an X-ray apparatus (X-Rad225, PRECISION, US) operating at a voltage of $225 \mathrm{kV}$ and a dose rate of $1 \mathrm{~Gy} / \mathrm{min}$.

All the irradiations were carried out at room temperature, and the control groups were subjected to sham irradiation.

\section{Cell survival assay}

Cell survival was detected with the colony formation assay as reported previously [22]. Cells in the logarithmic growth phase were irradiated with $\mathrm{CI}$ for $0,1,2,3,4$, and $6 \mathrm{~Gy}$, respectively. The irradiated cells were seeded into $60 \mathrm{~mm}$ cell culture dishes, with 3 parallels for each treatment group. After two weeks of culture, the medium was discarded, the adherent cells were stain with crystal violet solution for 30 minutes. The number of clones formed was counted (a clone was identified as a community with more than 50 cells), and the cell survival rate of each culture dish was calculated.

\section{Cytoplasmic calcium detection}

Calcium concentration in the cytoplasm was measured with Fluo-4, AM Fluo Calcium Indicators (Invitrogen, US) according to the manufacturer's instructions. The Fluo-4, AM ester stock solution was diluted to a final working concentration of $5 \mu \mathrm{M}$ with medium. Cells were incubated with the Fluo-4, AM ester for 30 minutes at $37^{\circ} \mathrm{C}$, then washed 3 times with PBS, incubated for another 30 minutes to ensure complete de-esterification of intracellular AM esters, and then detected via fluorescence microscopy or flow cytometry.

\section{Cell cycle assay}

Cells were collected and stained with a commercial kit according to the manufacturer's instructions (Beyotime, China). Cells were digested with EDTA-free trypsin, washed with pre-cooled PBS and collected by centrifugation, then fixed with pre-cooled $70 \%$ ethanol at $4^{\circ} \mathrm{C}$ for 24 hours. Then cells were collected by centrifugation and stained 500ul propidium iodide containing RNase A Dyeing solution, incubated for 30 minutes at $37^{\circ} \mathrm{C}$ in the dark, and detected by flow cytometry immediately. The cell cycle was analyzed with the Modfit LT software (version 3.1).

\section{Apoptosis assay}

Cells were stained with FITC Annexin V Apoptosis detection kit (BD Pharmingen, US) according to the manufacturer's instructions. Digested Cells were washed with pre-cooled PBS and resuspended in $1 \mathrm{ml}$ Binding Buffer, added $5 \mu \mathrm{l}$ of FITC Annexin V and $5 \mu \mathrm{l}$ PI, gently vortexed and incubated for 15 minutes at RT in the dark, and apoptosis was analyzed via flow cytometry immediately.

\section{DNA double-strand breaks}

Cells were pretreated and fixed with freshly prepared $4 \%$ paraformaldehyde for 10 minutes, and permeabilized with $0.25 \%$ Triton-X 100 for 10 minutes. Then, cells were blocked with 5\% BSA for 60 minutes, incubated with $\mathrm{\gamma H} 2 \mathrm{AX}$ primary antibody (2 hours) and fluorescent secondary antibody (45 minutes) in sequence at room temperature. The slides were stained with Hoechst 33342 and sealed with 20ul anti-quencher and inspected with a fluorescence microscope (OLYMPUS BX51).

\section{qRT-PCR}

Total RNA was extracted using TRIzol reagent (Invitrogen, US) according to the standard procedures [23] and concentration was measured with a microplate reader. cDNA was synthesized with riboSCRIPT Reverse Transcription Kit (RIBOBIO, China) according to the manufacturer's protocol. Quantitative real-time PCR was performed using QuantiNova SYBR Green PCR Kit (QIAGEN, Germany) and reaction samples were run on a QuantStudio5 qPCR system (ThermoFisher, US). The primers of qRT-PCR were synthesized by RIBOBIO (China). The $\mathrm{Ct}$ values for each gene were normalized to those of GAPDH, and the $2^{-\Delta \Delta C t}$ method was used for quantitative analysis of gene expression.

\section{Immunoblot analysis}

The whole proteins were extracted and conducted to SDS-PAGE as the standard procedure described previously [24]. The blots were transferred to PVDF membrane, blocked with blocking buffer (Beyotime, China) for $10 \mathrm{~min}$, incubated with primary antibodies at $4{ }^{\circ} \mathrm{C}$ overnight, then incubated with horseradish peroxidase-conjugated secondary antibodies for 1 hour and finally visualized by the 
enhanced chemiluminescence (ECL) procedure. The relative expression level of each protein was compared with the individual band density for beta-Actin and normalized by the corresponding control band.

\section{Statistical analysis}

Cell count and measurement of blots density were performed with the ImageJ software (v1.52). Data were presented as mean \pm standard deviation (SD). The variance homogeneity test and mean ANOVA analysis was performed with the IBM SPSS software (v21.0). Differences were considered significant and extremely significant when $p<0.05\left(^{*}\right)$ and $p<0.01\left({ }^{* *}\right)$, respectively.

\section{Results}

\section{ER stress induced by $\mathbf{C l}$ irradiation}

The cytosolic $\mathrm{Ca}^{2+}$ in HepG2 cells increased significantly 4 hours after irradiation with $\mathrm{CI}$ and returned to the basal level 24 hours after irradiation. $\mathrm{CI}$ irradiation also caused continuous $\mathrm{Ca}^{2+}$ release in
Hep3B cells and the release maintained until 24 hours after irradiation. However, the CI irradiation had no significant effect on the cytoplasmic $\mathrm{Ca}^{2+}$ concentration in PLC/PRF/5 cells (Fig. 1A, B and S1A).

The mRNA levels of Bip in the three HCC cell lines were up-regulated after irradiation, and the levels of PERK and ATF4 mRNAs in the downstream also increased in varying degrees. 4-PBA inhibited the up-regulation of Bip, PERK and ATF4 mRNA levels caused by irradiation (Fig. 1C, D and E). The autophagy inhibitor CQ did not reduce the levels of ER stress-related mRNA significantly.

CI irradiation alone did not change the expression of Bip in the three HCC cell lines, while the expression of PERK was up-regulated in HepG2 and $\mathrm{PLC} / \mathrm{PRF} / 5$ cells at $24 \mathrm{~h}$ and $48 \mathrm{~h}$, respectively. CI increased ATF4 in HepG2 cells at 48h while in Hep3B and PLC/PRF/5 cells at $24 \mathrm{~h}$. CI combined with sorafenib treatment further enhanced the expression of the above genes in HepG2 cells (Fig. 1F, G and H).
A

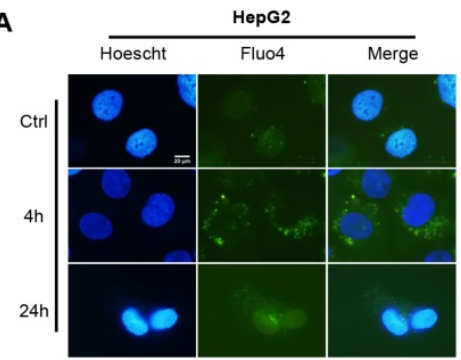

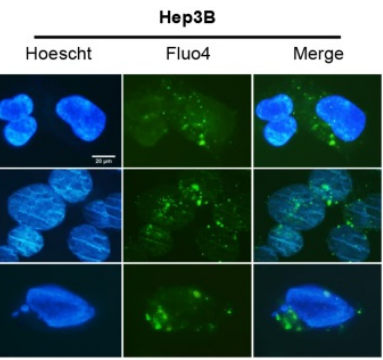

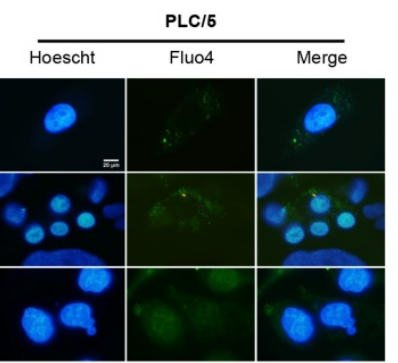

B

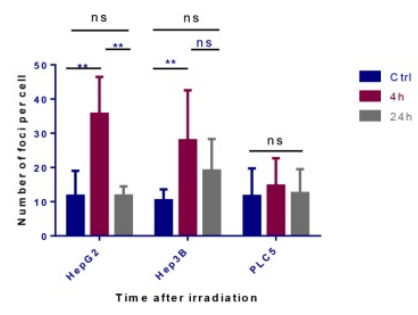

C

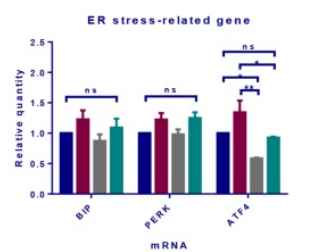

D

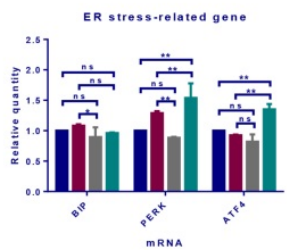

E

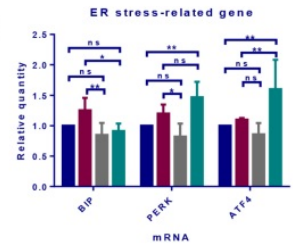

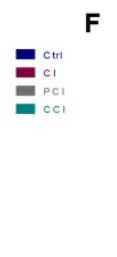

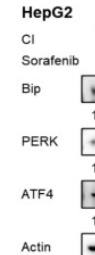

$\frac{24 \mathrm{~h}}{-+}$

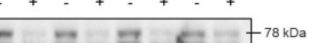
$\begin{array}{llllllll}1.0 & 0.1 & 0.8 & 0.2 & 1.0 & 0.2 & 0.9 & 0.5\end{array}$ - - - - - - - $140 \mathrm{kDa}$ $\begin{array}{llllllll}1.0 & 1.6 & 2.0 & 2.5 & 1.0 & 0.7 & 0.8 & 0.7\end{array}$

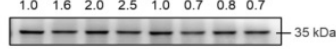

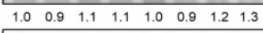
Actin $-(---\cdots-\cdots$

G нерзв

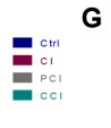

Sep3B

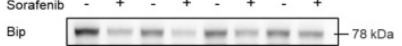

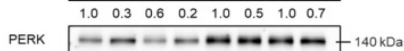

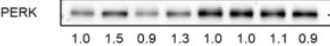

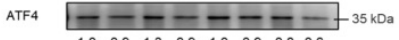

Actin $\quad \begin{array}{llllllll}1.0 & 0.9 & 1.3 & 0.9 & 1.0 & 0.9 & 0.8 & 0.6 \\ & - & - & - & - & - & - & -\end{array}$

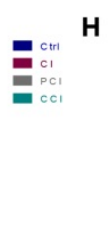

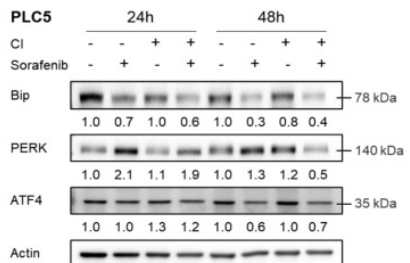

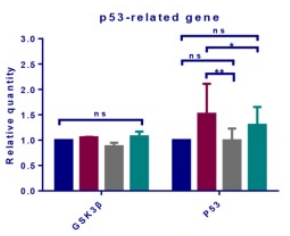

mRNA

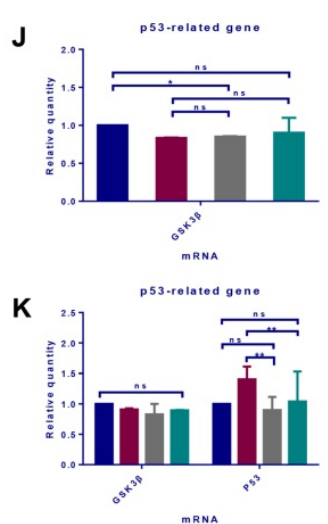

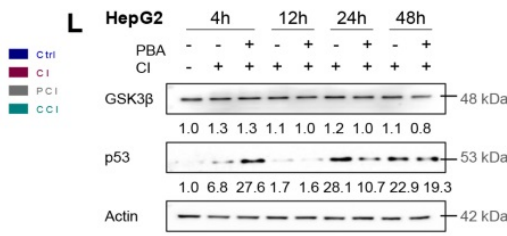

M

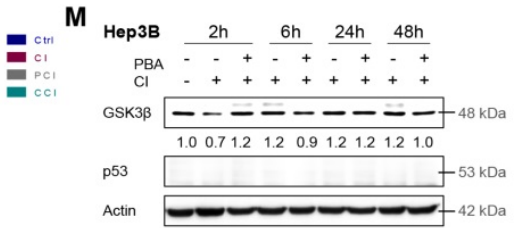

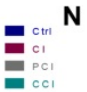

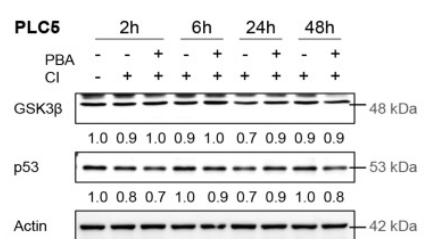

Figure 1. High-LET carbon ions $(\mathrm{Cl})$ promoted the expression of $\mathrm{p} 53$ and induced ER stress in HCC cell lines. HepG2, Hep3B and PLC/PRF/5 cells were pre-treated with $10 \mu M$ $\mathrm{CQ}$ for $4 \mathrm{~h}, 0.5 \mathrm{mM}$ 4-PBA for $12 \mathrm{~h}, 10 \mu \mathrm{M}$ sorafenib for $12 \mathrm{~h}$, respectively. Irradiations were conducted with carbon ions (LET = $50 \mathrm{keV} / \mu \mathrm{m}$ ) at a dose of $2 \mathrm{~Gy}$. The cytosolic calcium ions (Fluo-4, AM labeled) fluctuated in the three $\mathrm{HCC}$ cell lines after irradiation (A, B). The mRNA and protein levels of ER stress-related genes in HepG2 (C, F), Hep3B $(D, G)$ and PLC/PRF/5 (E, H) cells after irradiation. The levels of $\mathrm{P} 53$ mRNA and protein in HepG2 (I, L), Hep3B (J, M) and PLC/PRF/5 (K, N) cells after irradiation. (Ctrl: control; $\mathrm{PCl}$ : Cl treatment combined with 4-PBA; CCl: Cl treatment combined with $\mathrm{CQ}$. ns: not significant, *: $p<0.05, * *: p<0.01)$. 
A

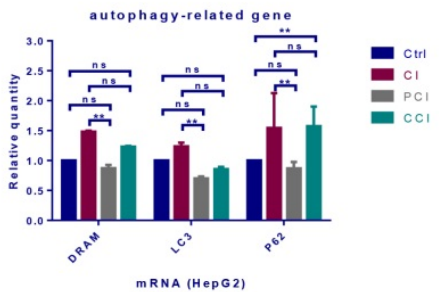

B

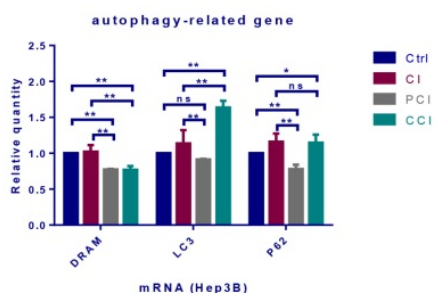

C

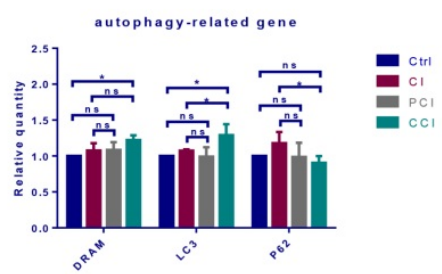

D
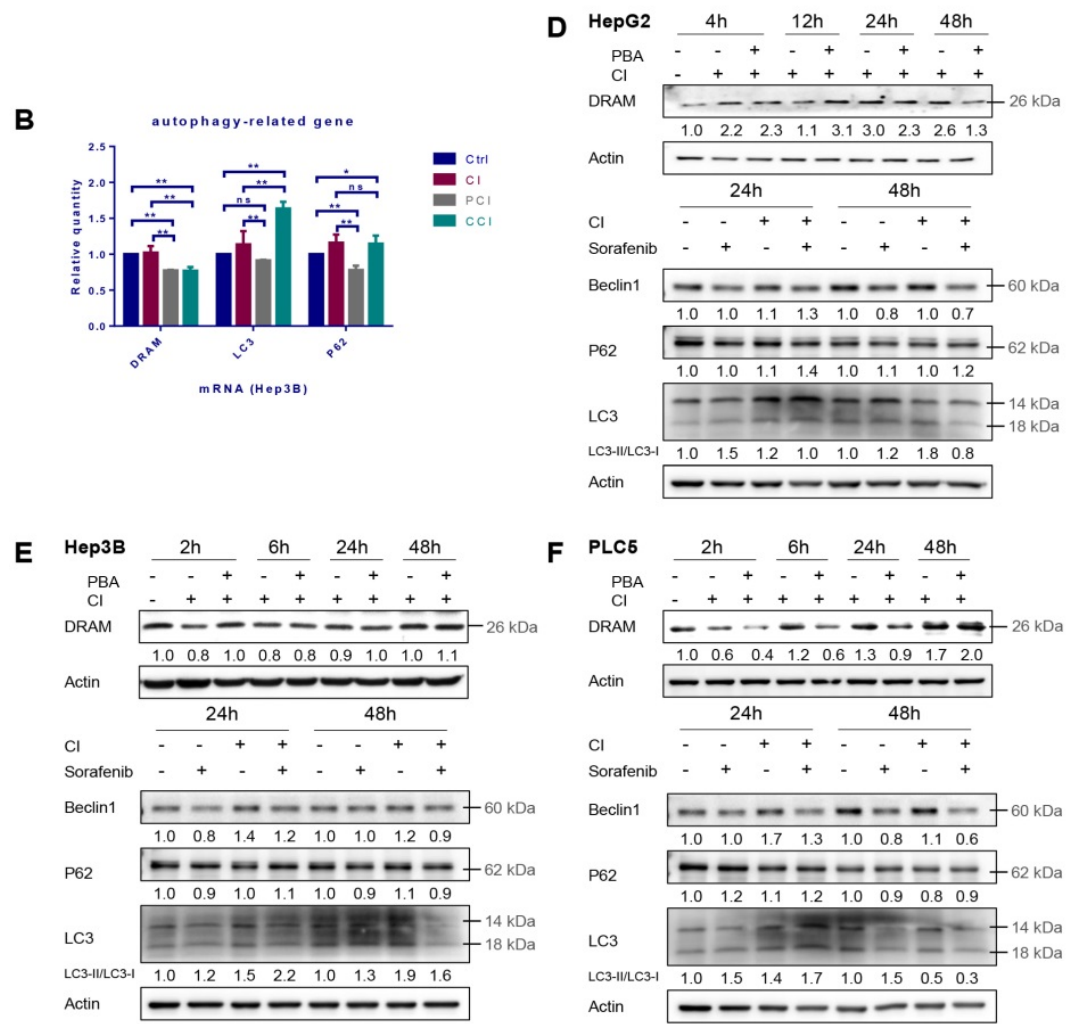

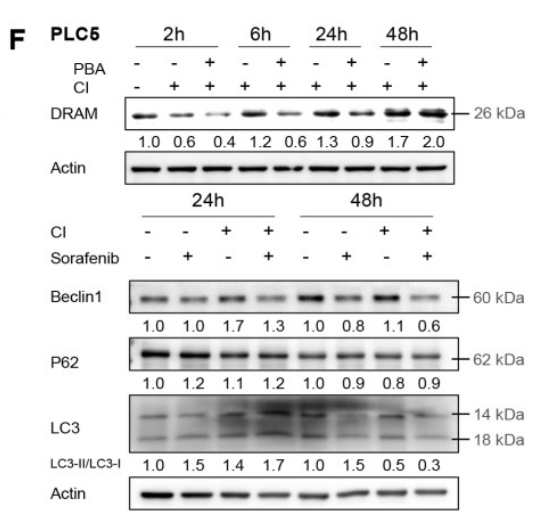

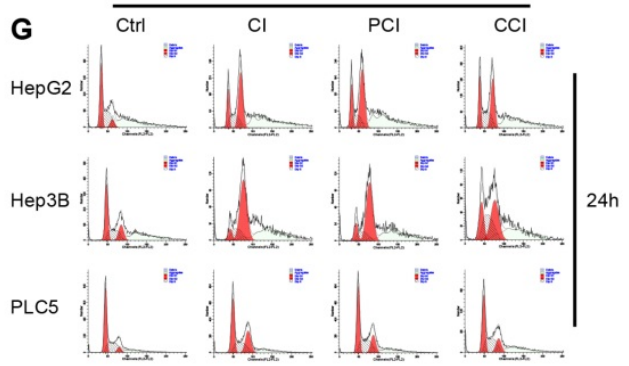

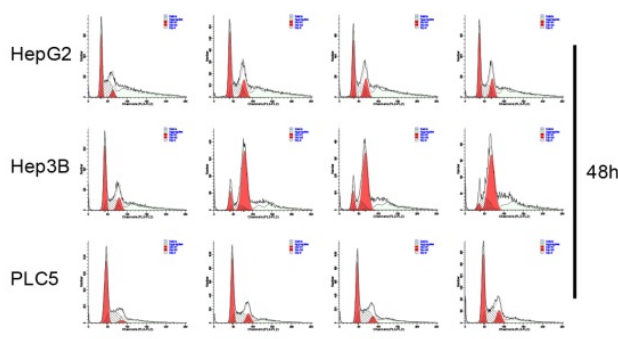

H

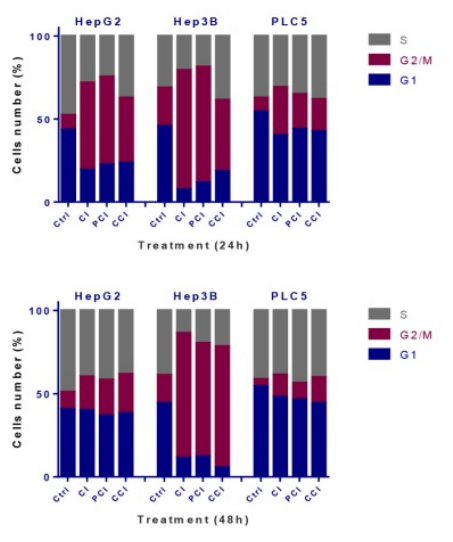

Figure 2. Autophagy and cell cycle arrest induced by carbon ion irradiation. HepG2, Hep3B and PLC/PRF/5 cells were pre-treated with $10 \mu M$ CQ for 4h, $0.5 \mathrm{mM} 4-\mathrm{PBA}$ for $12 \mathrm{~h}$, $10 \mu \mathrm{M}$ sorafenib for $12 \mathrm{~h}$, respectively. Irradiations were conducted with carbon ions (LET = $50 \mathrm{keV} / \mu \mathrm{m}$ ) at a dose of $2 \mathrm{~Gy}$. The mRNA and protein levels of autophagy-related genes in HepG2 (A, D), Hep3B (B, E) and PLC/PRF/5 (C, F) cells after irradiation. Cell cycle arrest induced by carbon ion irradiation in the three HCC cell lines (G, H). (Ctrl: control; PCl: $\mathrm{Cl}$ treatment combined with 4-PBA; $\mathrm{CCl}$ : Cl treatment combined with CQ. ns: not significant, *: $p<0.05, * *: p<0.01)$.

CI irradiation up-regulated the mRNA expression level of TP53 gene in HepG2 and PLC/PRF/5 cells (Fig. 1I and K), and the expression of p53 in HepG2 cells increased significantly post-irradiation, while there was no significant effect on the expression of p53 in PLC/PRF/ 5 cells and no p53 was detected in Hep3B cells (Fig. 1J, and L-N).

\section{Autophagy induced by $\mathrm{Cl}$ irradiation}

The mRNA levels of DRAM, LC3 and p62 were up-regulated after $\mathrm{CI}$ irradiation in all the three HCC cell lines, while 4-PBA combined with CI irradiation reduced the mRNA levels of these autophagy-related genes (Fig. 2A, B and C). The protein levels of DRAM, Beclin-1 and LC3 after irradiation showed a time-dependent trend. Specifically, DRAM and LC3 cleavage increased continuously after irradiation, Beclin- 1 increased by CI combined with sorafenib at 24h after treatment in HepG2 cells (Fig. 2D). Irradiation did not augment the expression of DRAM in Hep3B cells, but increased the expression of Beclin-1 and LC3 cleavage at $24 \mathrm{~h}$ and $48 \mathrm{~h}$ (Fig. 2E). Moreover, in PLC/PRF/ 5 cells, CI irradiation induced the up-regulation of DRAM at $48 \mathrm{~h}$, while the expression of Beclin-1 and the cleavage of LC3 were enhanced at 24h (Fig. 2F). Notably, p62 increased while LC3 cleavage decreased in HepG2 cells after CI combined with sorafenib treatment. P62 had no significant change in trend after treatment in Нер3B and PLC/PRF/ 5 cells. In addition, the presence of a double-layer membrane structure containing inclusions in the irradiated cells, namely autophagic vacuoles, was observed with transmission electron microscopy (TEM) (Fig. 4B).

\section{Cell cycle arrest and apoptosis induced by $\mathrm{Cl}$ irradiation}

Twenty-four hours after irradiation, the $G_{2} / M$ phase arrest occurred in all three HCC cell lines. Hep3B cells presented the most significant cell cycle 
arrest while the level of cell cycle arrest in PLC/PRF/5 cells was the least. Forty-eight hours after irradiation, the cycle arrest of Hep3B cells did not alleviate at all, whereas the cycle arrest recovered to a lower level in HepG2 and PLC/PRF/ 5 cells (Fig. $2 \mathrm{G}$ and $\mathrm{H}$ ).

CI irradiation caused DNA DSBs in all three HCC cell lines. The average $\gamma \mathrm{H} 2 \mathrm{AX}$ foci number per cell in HepG2 cells was significantly higher than those in Hep3B and PLC/PRF/5 cells at 2 hours after irradiation. Twenty-four hours after irradiation, the average number of foci in all three cell lines decreased, and the number of foci in Hep3B cells was significantly lower than those in the other two cell lines (Fig. 3A and B).

Twenty-four hours after CI irradiation, the apoptotic rate of HepG2 cells was significantly higher than those of the other two cell lines. At $48 \mathrm{~h}$ after irradiation, the apoptotic rates of HepG2 and PLC/PRF/5 cells increased compared with $24 \mathrm{~h}$ post-irradiation, while the apoptotic rate of Hep3B cells remained at a low level (Fig. 3C and D).

At the same dose, the cellular sensitivity to $\mathrm{CI}$ irradiation became low for TP53 wild-type HepG2, TP53-null Hep3B and TP53 mutant PLC/PRF/5 cells in turn (Fig. 3E).

CI irradiation up-regulated the expression of PUMA, NOXA and Bcl-2, and PBA inhibited the phosphorylation of $\mathrm{Bcl}-2$ in HepG2 cells. In Hep3B cells, CI irradiation up-regulated the expression of PUMA, down-regulated the level of pBcl-2 2-6h post-irradiation, and PBA inhibited the phosphorylation of $\mathrm{Bcl}-2$, resulting in Caspase-3 cleavage and cellular apoptosis. In PLC/PRF/ 5 cells, CI irradiation down-regulated the expression of PUMA and NOXA, while PBA inhibited the phosphorylation of Bcl-2, which also increased the cleavage of Caspase-3 and apoptosis 24-48h post-irradiation (Fig. 3F-H).
A
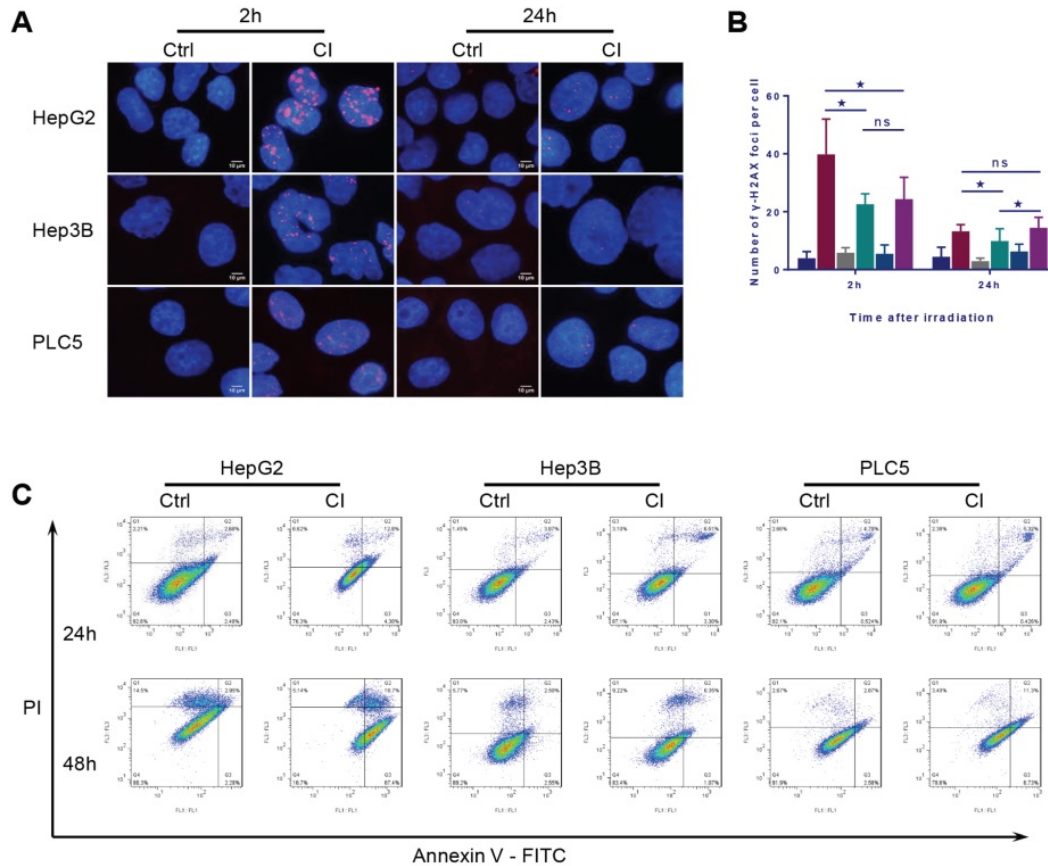

D

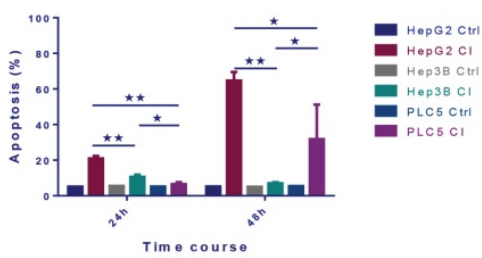

B

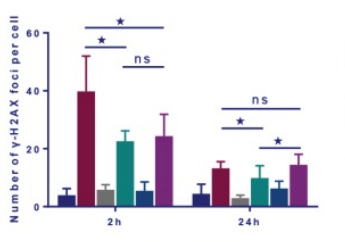

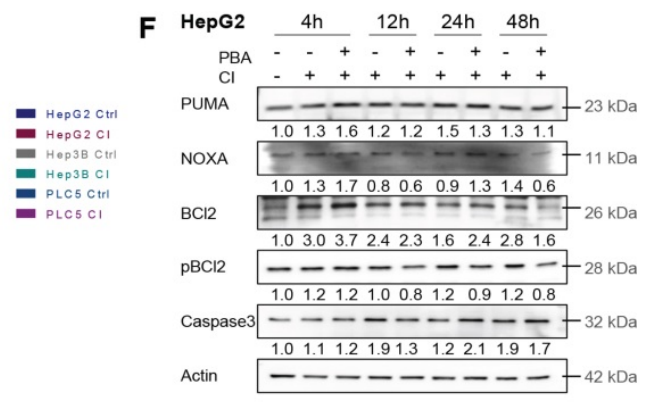

G

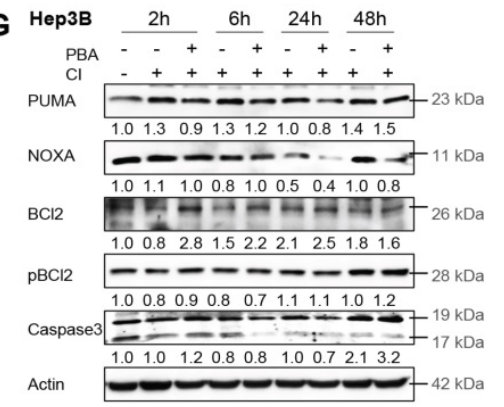

H

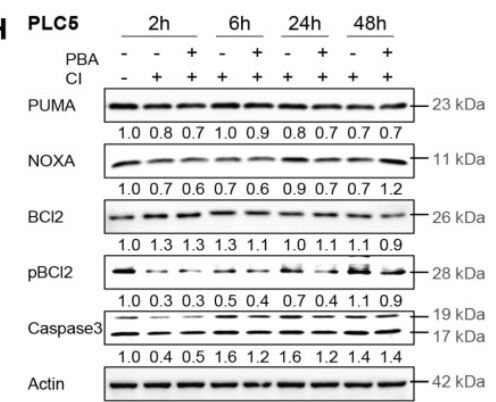

Figure 3. Apoptosis induced by carbon ion irradiation. HepG2, Hep3B and PLC/PRF/5 cells were pre-treated with $0.5 \mathrm{mM} 4$-PBA for $12 \mathrm{~h}$, and then irradiations were conducted with carbon ions (LET $=50 \mathrm{keV} / \mathrm{um}$ ) at a dose of $2 \mathrm{~Gy}$. DNA double-strand breaks (Labeled with $\mathrm{YH} 2 \mathrm{AX}$ antibody) in three HCCs after irradiation (A, B). The apoptosis rates in the three $\mathrm{HCC}$ cell lines after irradiation (C, D). The clonogenic survival data of the three $\mathrm{HCC}$ cell lines after irradiation (E). $\mathrm{HCC}$ cells were irradiated with carbon ions at a dose of $0,1,2,3,4$ and $6 \mathrm{~Gy}$, respectively. The expression levels of apoptosis-related proteins in HepG2 (F), Hep3B (G) and PLC/PRF/5 (H) after irradiation. 


\section{Ferroptosis induced by $\mathrm{Cl}$ irradiation combined with sorafenib treatment}

By using Mito-Tracker Red CMXRos probe to label mitochondria, the images of laser scanning confocal microscopy (LSCM) showed that the mitochondria changed from diffuse state into dot state after CI combined with sorafenib treatment, and the fluorescence intensity increased compared with the control group (Fig. 4A). The results of transmission electron microscopy (TEM) showed that CI combined with sorafenib treatment increased mitochondrial membrane density and decreased mitochondrial crest (Fig. 4B). CI irradiation caused an increase in the level of lipid peroxidation product malondialdehyde (MDA), and CI combined with sorafenib treatment further increased the level of MDA significantly (Fig. $4 \mathrm{C})$.

Irradiation had no significant effect on the mRNA level of SLC7A11 in HepG2 and PLC/PRF/5 cells, but 4-PBA or CQ reduced the mRNA level of SLC7A11 (Fig. 4D and F). In Hep3B cells, irradiation slightly increased the mRNA level of SLC7A11. 4-PBA reduced the mRNA level, but $C Q$ significantly increased the mRNA level of SLC7A11 (Fig. 4E).

The expression level of $\mathrm{xCT}$ (encoded by SLC7A11) was down-regulated by sorafenib and further decreased by $\mathrm{CI}$ combined with sorafenib treatment in all the three HCCs (Fig. 4G, H and I). The level of GPX4 was slightly down-regulated by CI combined with sorafenib treatment in HepG2 and Hep3B cells, but not in PLC/PRF/5 cells. CI irradiation increased the expression of PTGS2 at 24h and $48 \mathrm{~h}$ and even the combined treatment further, and increased the expression of $4-\mathrm{HNE}$ at $48 \mathrm{~h}$ in HepG2 cells. In comparison, CI combined with ferroptosis inhibitor Ferrostatin-1 treatment up-regulated the level of $\mathrm{xCT}$ and GPX4, while decreased PTGS2 in HepG2 cells (Fig. S1B). In Hep3B cells, CI irradiation up-regulated the level of PTGS2 and $4-\mathrm{HNE}$ at $48 \mathrm{~h}$, while the combined treatment decreased PTGS2 and 4-HNE at 24h but increased 4-HNE at $48 \mathrm{~h}$. In PLC/PRF/5 cells, CI irradiation up-regulated the level of PTGS2 and 4-HNE at $24 \mathrm{~h}$ and $48 \mathrm{~h}$. The combined treatment increased PTGS2 at $24 \mathrm{~h}$ and $4-\mathrm{HNE}$ at $24 \mathrm{~h}$ and $48 \mathrm{~h}$.

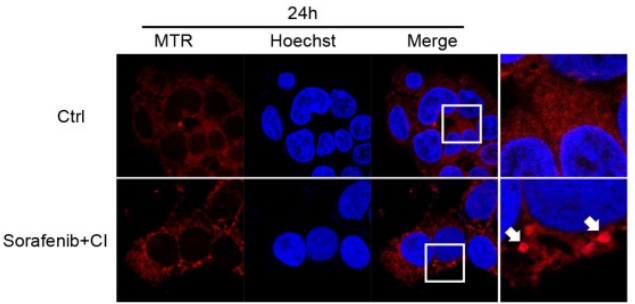

C

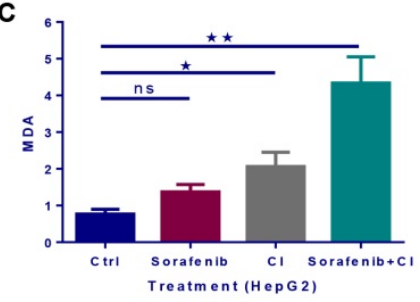

D

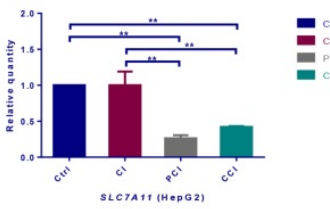

E
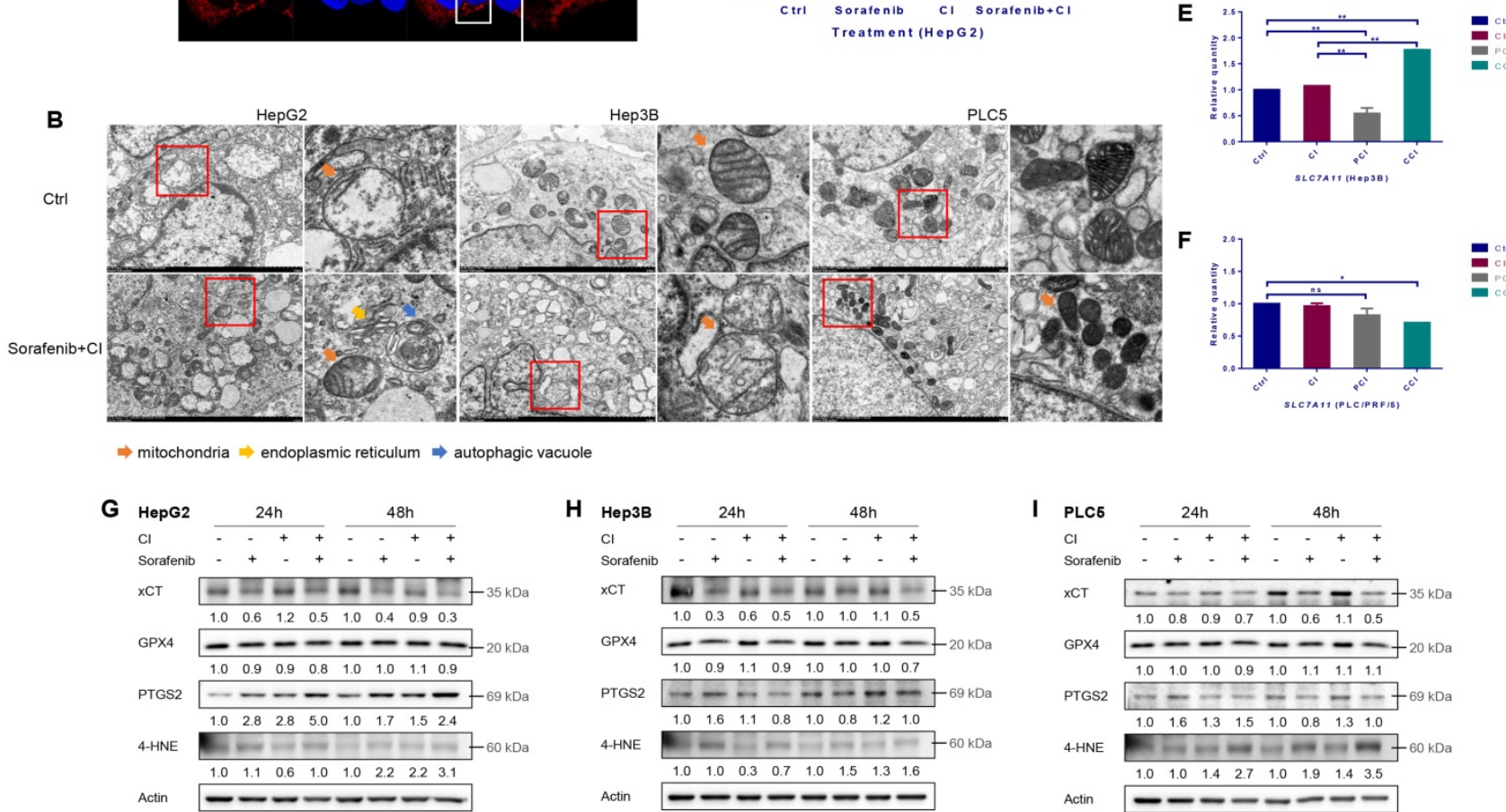

Hep3B
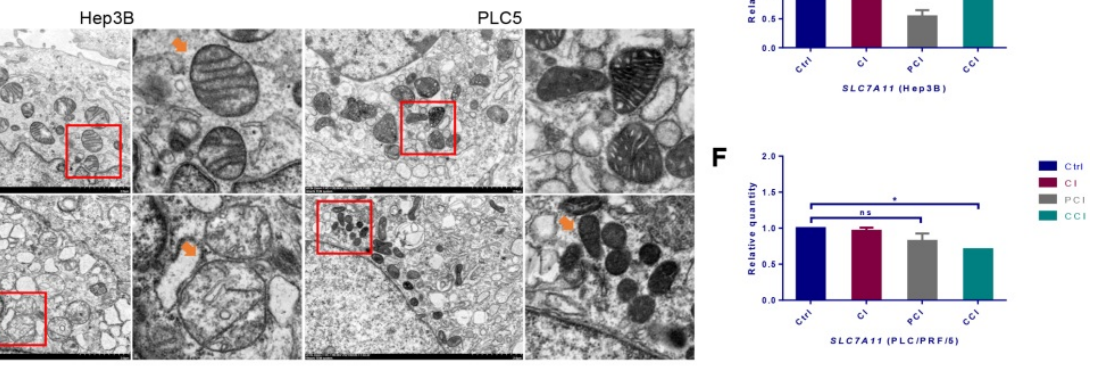
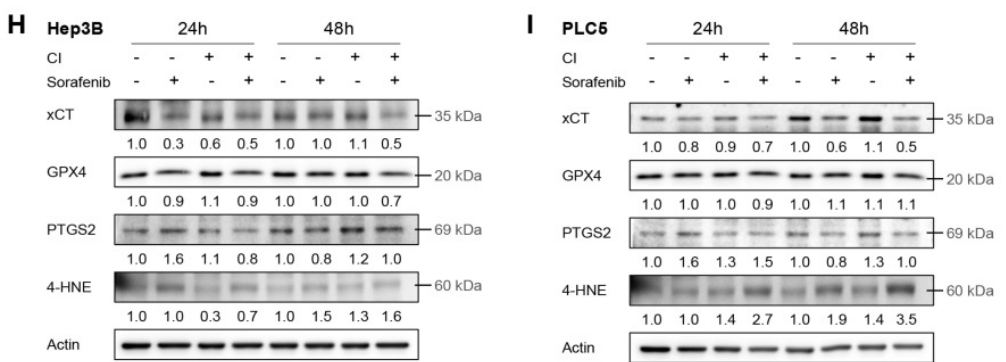

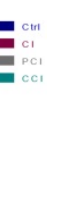

를

Figure 4. Ferroptosis induced by carbon ion irradiation. HepG2, Hep3B and PLC/PRF/5 cells were pre-treated with $10 \mu M$ CQ for $4 \mathrm{~h}, 0.5 \mathrm{mM} 4-\mathrm{PBA}$ for $12 \mathrm{~h}, 10 \mu \mathrm{M}$ sorafenib for $12 \mathrm{~h}$, respectively. Irradiations were conducted with carbon ions (LET $=50 \mathrm{keV} / \mu \mathrm{m}$ ) at a dose of $2 \mathrm{~Gy}$. (A) Representative morphological characteristics of mitochondria were observed using laser confocal microscopy. (B) Representative characteristics of ferroptosis and autophagic vacuoles were examined with a transmission electron microscope. (C) MDA level in HepG2 cells after carbon ion irradiation. The mRNA levels of SLC7A Il in HepG2 (D), Hep3B (E) and PLC/PRF/5 (F) cells after irradiation. The expression levels of ferroptosis-related proteins in HepG2 (G), Hep3B (H) and PLC/PRF/5 (I) cells after irradiation. (Ctrl: control. ns: not significant, *: $p<0.05, * *: p<0.01$ ). 


\section{Interference of TP53 expression affected the radiosensitivity of Hep $\mathbf{G} 2$ cells}

Protein expression and mRNA transcription of p53 were significantly reduced 48 hours after TP53 was inhibited by siRNA (Fig. 5A and B). The cellular apoptosis induced by irradiation after interference was significantly lower than that in the irradiation alone group (Fig. 5C and D). Besides, after the inhibition of TP53, CI irradiation caused more significant $G_{2} / M$ phase arrest and increased the proportion of $S$ phase cells (Fig. 5E and F). Inhibiting TP53 reduced the sensitivity of HepG2 cells to CI radiation (Fig. 5G). The interference of TP53 had no significant effect on the transcription of Bip, but slightly increased the mRNA level of PERK and ATF4. Inhibition of TP53 also up-regulated the transcription of autophagy-related LC3 and p62, together with the mRNA expression level of the ferroptosis-related gene SLC7A11 (Fig. 5H-K).

\section{Discussion}

damage, and is also one of the characteristic phenomena of ER stress [25]. The present study showed that different TP53 statuses lead to different degrees of $\mathrm{Ca}^{2+}$ release caused by CI irradiation. Studies have demonstrated that UPR plays a vital role in the treatment of HCCs by regulating the downstream pathways to favor pro-survival or pro-death signaling [26]. The mRNA expression levels of Bip, PERK and ATF4 were up-regulated by CI irradiation in the three HCC cell lines, and the translation of these mRNAs also increased, suggesting that ER stress was induced by high-LET radiation. PERK, often used as a tumor marker, indicates a higher tumor grade and a poor prognosis [27]. Inhibiting the activity of PERK can improve the radiosensitivity of tumor cells both in vitro and in vivo $[28,29]$. Therefore, PERK might be used as a potential therapeutic target, and the development of new drugs or exploring new mechanisms of existing clinical drugs targeting PERK will have significant therapeutic benefits against cancer.

$\mathrm{Ca}^{2+}$ fluctuation is considered as a sign of ER

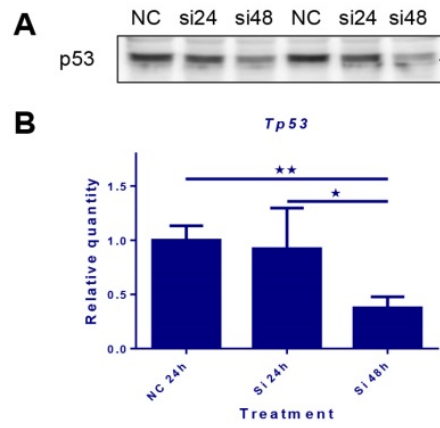

D
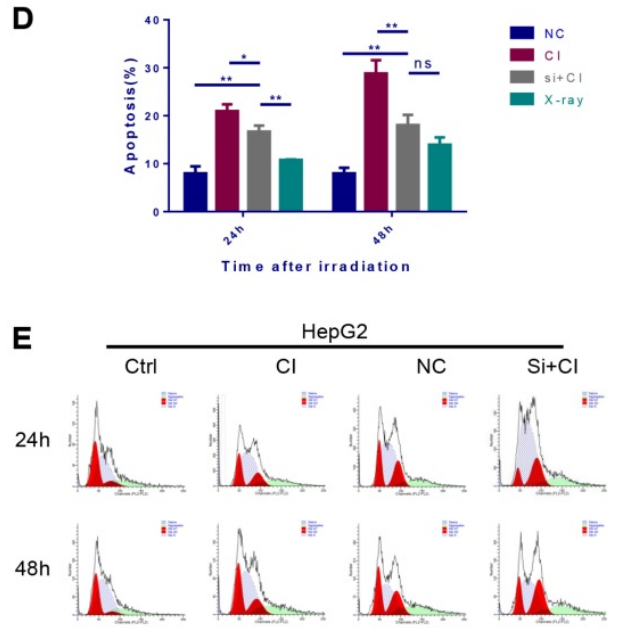

\section{C}

$$
\text { PI }
$$
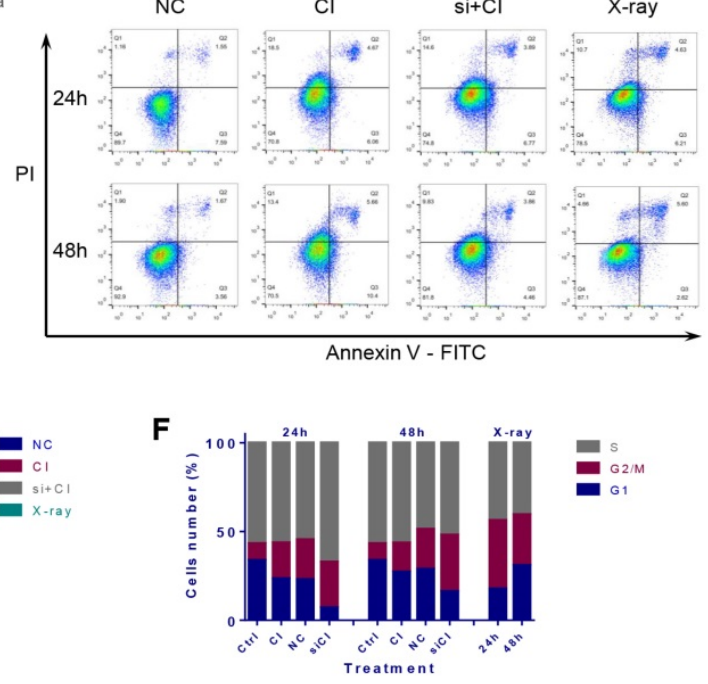

G

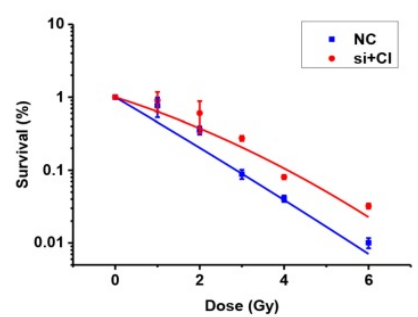

H

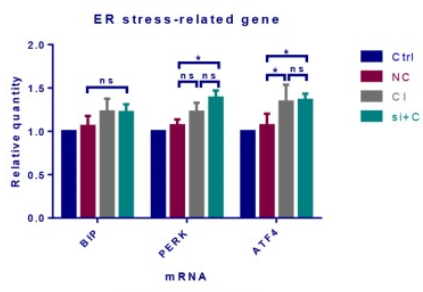

I

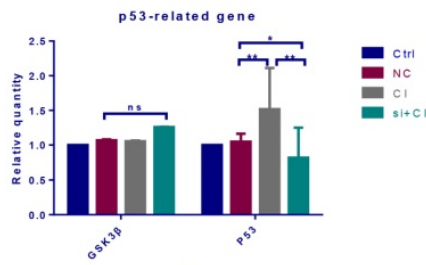

J

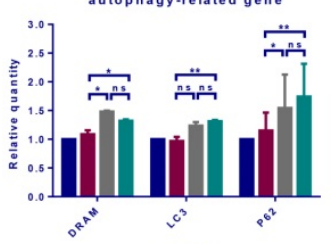

$\mathbf{E}_{\mathrm{Nc}}^{\mathrm{ctr}}$

$\mathbf{K}$

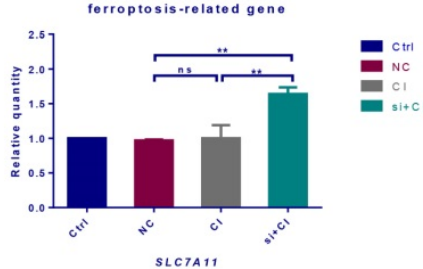

Figure 5. Interference of TP53 affected the radiosensitivity of HepG2 cells. HepG2 cells were transfected with TP53-siRNA for 48h, and irradiations were conducted with carbon ions (2Gy) or X-rays (4Gy). The expression level of TP53 gene in HepG2 cells after siRNA interference (A, B). The apoptosis rate of HepG2 cells caused by carbon ion irradiation after TP53 interference (C, D). The cell cycle arrest of HepG2 cells caused by carbon ion irradiation after TP53 interference (E, F). The clonogenic survival data of HepG2 cells after TP53 interference (G). In the case of TP53 interference, the levels of mRNA related to ER stress, p53, autophagy and ferroptosis in HepG2 cells after irradiation $(\mathrm{H}-\mathrm{K})(\mathrm{Ctrl}$ : control; NC: negative control; si $+\mathrm{Cl}$ : Cl treatment combined with siRNA. ns: not significant, $*: p<0.05, * *: p<0.01)$. 


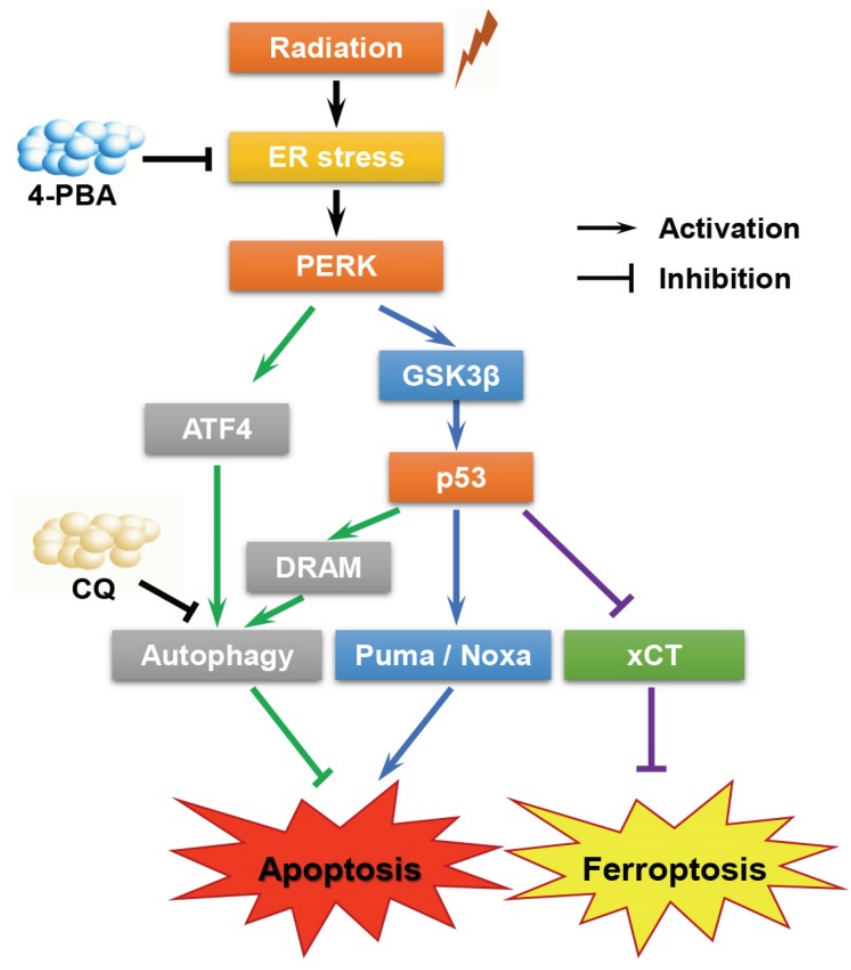

Figure 6. A proposed model of the molecular mechanisms by which PERK regulates the sensitivity of HCC cells with different TP53 statuses to high-LET carbon ions.

Autophagy plays a "double-edged sword" role in both cytotoxicity and cytoprotection [30-32]. DRAM is a key molecule for p53-activated autophagy. We confirmed that PERK and p53 signals jointly induced autophagy in HepG2 cells. Autophagy was induced by the PERK-ATF4 signaling in Hep3B and PLC/PRF/ 5 cells, so 4-PBA reduced the mRNA levels of autophagy-related genes in the above two HCC cells. CQ did not reduce the levels of ER stress-related mRNA significantly, indicating that the autophagy signal was in a relatively downstream position of the ER stress signaling network. These results are consistent with the findings that radiation-induced protective autophagy helps to clean the aggregated unfolded proteins, assists the quality control of ER proteins, and finally promotes the survival of tumor cells in the previous studies $[19,30]$.

The pro-death signal of UPR will eventually converge on the endogenous apoptotic pathway. PUMA and NOXA are two typical Bcl-2 family proteins regulated by $\mathrm{p} 53$. By up-regulating the expression of the pro-apoptotic PUMA and NOXA, the Caspase-3 is cleaved, and p53-dependent apoptosis is executed. Compared with HepG2 cells, there were fewer DNA DSBs and lower apoptosis rates in TP53-null Hep3B cells. This might be due to the fact that radiation caused a slight up-regulation of PUMA expression and Caspase- 3 cleavage. The extent of DNA DSBs and apoptosis in PLC/PRF/ 5 cells was between the other two HCC cell lines. Probably CI irradiation inhibited the expression of the anti-apoptotic pBcl-2 and promoted Caspase-3 cleavage. The clonogenic survival data were in line with the results above. These results jointly confirmed that the sensitivity of HepG2 cells to high-LET CI radiation was higher than those of the other two HCC cell lines.

The microscopic imaging results revealed that HCCs after CI combined with sorafenib treatment exhibited shrunken mitochondria with increased membrane density and decreased crest, a morphological feature of ferroptosis. CI irradiation caused an increase in the level of lipid peroxidation product MDA, and $\mathrm{CI}$ combined with sorafenib treatment further increased the level of MDA significantly. These results indicated that CI irradiation promoted the level of lipid peroxidation and induced the morphological characteristics of ferroptosis in the HCC cell lines.

$\mathrm{xCT}$ is the light chain subunit of the cystine-glutamate reverse transport system $X_{c^{-}}$, which can transport glutamate to extracellular space and extracellular cystine into cells [33]. Inhibiting the $\mathrm{X}_{\mathrm{c}}$ system reduces intracellular GSH, leading to the accumulation of lipid reactive oxygen species, and ultimately results in ferroptosis, which is a unique cell death mode different from apoptosis and necrosis [34-36]. Previous studies have shown that p53 can promote ferroptosis by transcriptionally suppressing SLC7A11 or promoting SAT1 and GLS2, and can also suppress ferroptosis by directly inhibiting DPP4 activity or inducing CDKN1A/p21 expression [37, 38]. Sorafenib is a multi-kinase inhibitor of Raf- 1 and B-Raf, which can simultaneously inhibit a variety of intracellular kinases involved in tumor cell signal transduction, angiogenesis and apoptosis, and exert anti-tumor effects. Sorafenib can also induce ferroptosis by inhibiting system $\mathrm{X}_{\mathrm{c}}{ }^{-}$activity. In this study, CI irradiation alone had a limited effect on the mRNA and protein levels of SLC7A11 in all the three HCC cell lines, while CI combined with sorafenib treatment significantly reduced the expression of $\mathrm{xCT}$. These results indicated that the combined treatment of $\mathrm{CI}$ and sorafenib initiated extrinsic ferroptosis by inhibiting cell membrane transporters $[39,40]$.

Glutathione peroxidase (GPX4) uses glutathione to convert lipid hydroperoxides into non-toxic lipid alcohols, thereby reducing lipid peroxidation and inhibiting ferroptosis. In this study, we found that CI had no significant effect on GPX4, indicating that GPX4 did not participate in ferroptosis caused by CI irradiation. In other words, CI did not activate ferroptosis through the intrinsic pathway of blockade of intracellular antioxidant enzymes. 
PTGS2 serves as another marker of ferroptosis induction in preclinical models. CI irradiation increased the expression of PTGS2 in the three HCC cell lines to varying degrees, and CI combined with sorafenib treatment further increased the PTGS2 level. 4-HNE and MDA are typical products of lipid peroxidation, they usually act as specific ferroptosis-related damage-associated molecular patterns (DAMPs) to induce ferroptosis-specific inflammatory and immune responses [41]. We found that the expression of 4-HNE was up-regulated by CI irradiation and further increased by $\mathrm{CI}$ combined with sorafenib in all three HCCs. In HepG2 cells, the level of MDA showed the same trend as 4-HNE. These results indicated that $\mathrm{CI}$ irradiation combined with sorafenib treatment inhibited the ROS scavenging system $X_{c}^{-}$pathway, thus leading to the accumulation of lipid peroxidation products, which might eventually lead to ferroptosis and help to enhance the inhibitory effect of high-LET CI irradiation on HCC cells.

We found that inhibition of radiation-induced ER stress mainly caused HepG2 cells to undergo mixed-type RCD of both apoptosis and ferroptosis; however, down-regulation of $\mathrm{xCT}$ indicated that ferroptosis with a low level of apoptosis might occur in Hep3B and PLC/PRF/5 cells, demonstrating that ER stress could relieve apoptosis and ferroptosis, and promoting cell survival. Previous studies have shown that activation of autophagy is a common event during ferroptosis, but its role is still inconclusive [35, 42]. In this study, inhibition of autophagy resulted in a decrease of SLC7A11 transcription in HepG2 and PLC/PRF/5 cells. This proves that autophagy could alleviate ferroptosis and promote cell survival in TP53-wild cells. After CQ treatment, the mRNA level of SLC7A11 in Hep3B cells increased significantly, indicating that bulk autophagy could promote ferroptosis in TP53-null cells.

There have been many studies on the mechanism of TP53 in regulating radiosensitivity, and it has been generally believed that the absence of TP53 reduces the sensitivity to conventional radiations such as X-rays. Our results showed that inhibition of TP53 reduced apoptosis induced by $\mathrm{CI}$ irradiation and promoted cell survival. We also found that HepG2 cells showed an easy-to-recover $\mathrm{G}_{2} / \mathrm{M}$ phase arrest after irradiation, and inhibiting TP53 led to a more obvious and difficult-to-recover $G_{2}$ / $M$ phase arrest. This is consistent with the phenomenon observed for cell cycle arrest in TP53-null Hep3B cells. Interference of TP53 significantly up-regulated the mRNA expression level of SLC7A11, suggesting that p53 might promote ferroptosis by transcriptionally suppressing SLC7A11. This is in line with the conclusion that activation of TP53 significantly reduces the expression of SLC7A11 and knockout of TP53 eliminates the inhibitory effect on SLC7A11 [43-45]. Collectively, TP53 was involved in cell cycle regulation, apoptosis promotion and ferroptosis induction, which jointly regulated the response of HCC cells to ionizing radiation.

In summary, the present study confirmed that high-LET CI radiation could induce ER stress in HCC cell lines. On the one hand, PERK promoted autophagy and maintained cell survival under ER stress via regulating ATF4 expression; on the other hand, PERK regulated p53 expression, and the latter either induced autophagy by up-regulating DRAM, or directly promoting apoptosis through the mitochondrial pathway or promoting ferroptosis via inhibiting the transcription and translation of SLC7A11. Besides, p53 also participated in cell cycle regulation. The above-mentioned factors jointly determined the fate of HCC cells (Fig. 6). Moreover, apoptosis and ferroptosis are synergetic cell death modes regulated by TP53. High-LET CI irradiation combined with sorafenib treatment could lead to the induction of a mixed-type RCD including both apoptosis and ferroptosis in HepG2 cells. This might be one of the reasons why the radiosensitivity of TP53 wild-type HepG2 cells was higher than that of Hep3B and PLC/PRF/ 5 cells. Due to such a central role of TP53 in cell signaling, its deletion or mutation directly increases cellular radioresistance. Therefore, our work may shed light on the potential therapeutic implication of high-LET CI radiotherapy combined with PERK targeted drugs to implement personalized and precise treatment of HCCs with different TP53 statuses.

\section{Abbreviations}

4-PBA: 4-phenylbutyric acid; ATF6: activating transcription factor 6; CI: carbon ions; CQ: chloroquine; ER: endoplasmic reticulum; HCC: hepatocellular carcinoma; IRE1: inositol requiring enzyme 1; LET: linear transfer energy; PERK: PKR-like ER kinase; RCD: regulated cell death; SLC7A11: solute carrier family 7 member 11; UPR: unfolded protein response.

\section{Supplementary Material}

Supplementary figure.

https://www.jcancer.org/v13p0669s1.pdf

\section{Acknowledgements}

This work was jointly supported by the National Natural Science Foundation of China (Grant Nos. 12005268, 11875299, 11705245), the National Key Research and Development Program of China (Grant 
No. 2018YFC0115702), the Key Deployment Project of the Chinese Academy of Sciences (Grant No. KFZD-SW-222), the Western Talents Program of the Chinese Academy of Sciences (Grant No. (2018) No.99), the Natural Science Foundation of Gansu Province (20JR5RA549) and the Foundation of the Ministry of Education Key Laboratory of Cell Activities and Stress Adaptations (lzujbky-2021-kb05).

\section{Ethics Statement}

This study did not include human participants, human data or human tissue.

\section{Author Contributions}

X.Z.: Conceptualization, Methodology, Investigation, Writing - Original Draft, Reviewing and Editing. B.L., X.L., P.L., P.Z., F.Y., T.Z., Y.K., W.C.: Investigation, analysis and interpretation of data. X.J.: Methodology, Supervision. Q.L.: Supervision, Writing - Review \& Editing. All authors read and approved the final manuscript.

\section{Competing Interests}

The authors have declared that no competing interest exists.

\section{References}

1. Walenta S, Mueller-Klieser W. Differential Superiority of Heavy Charged-Particle Irradiation to X-Rays: Studies on Biological Effectiveness and Side Effect Mechanisms in Multicellular Tumor and Normal Tissue Models. Frontiers in oncology. 2016; 6: 30.

2. Kamada T, Tsujii H, Blakely EA, Debus J, De Neve W, Durante M, et al. Carbon ion radiotherapy in Japan: an assessment of 20 years of clinical experience. The Lancet Oncology. 2015; 16: e93-e100.

3. Forner A, Reig M, Bruix J. Hepatocellular carcinoma. Lancet. 2018; 391: 1301-14.

4. Lin ZZ, Chou CH, Cheng AL, Liu WL, Chia-Hsien Cheng J. Radiosensitization by combining an aurora kinase inhibitor with radiotherapy in hepatocellular carcinoma through cell cycle interruption. International journal of cancer Journal international du cancer. 2014; 135: 492-501.

5. Qi WX, Fu S, Zhang Q, Guo XM. Charged particle therapy versus photon therapy for patients with hepatocellular carcinoma: a systematic review and meta-analysis. Radiotherapy and oncology : journal of the European Society for Therapeutic Radiology and Oncology. 2015; 114: 289-95.

6. Spychalski P, Kobiela J, Antoszewska M, Blazynska-Spychalska A, Jereczek-Fossa BA, Hoyer M. Patient specific outcomes of charged particle therapy for hepatocellular carcinoma - A systematic review and quantitative analysis. Radiotherapy and Oncology. 2019; 132: 127-34.

7. Nickoloff JA. Photon, light ion, and heavy ion cancer radiotherapy: paths from physics and biology to clinical practice. Annals of translational medicine. 2015; 3: 336.

8. Ron D, Walter P. Signal integration in the endoplasmic reticulum unfolded protein response. Nature reviews Molecular cell biology. 2007; 8: 519-29.

9. Bhat TA, Chaudhary AK, Kumar S, O'Malley J, Inigo JR, Kumar R, et al. Endoplasmic reticulum-mediated unfolded protein response and mitochondrial apoptosis in cancer. Bba-Rev Cancer. 2017; 1867: 58-66.

10. Shi W, Chen Z, Li L, Liu H, Zhang R, Cheng Q, et al. Unravel the molecular mechanism of XBP1 in regulating the biology of cancer cells. Journal of Cancer. 2019; 10: 2035-46.

11. Wang M, Kaufman RJ. Protein misfolding in the endoplasmic reticulum as a conduit to human disease. Nature. 2016; 529: 326-35.

12. Hetz C, Papa FR. The Unfolded Protein Response and Cell Fate Control. Molecular cell. 2018; 69: 169-81.
13. Maurel M, McGrath EP, Mnich K, Healy S, Chevet E, Samali A. Controlling the unfolded protein response-mediated life and death decisions in cancer. Seminars in cancer biology. 2015; 33: 57-66.

14. Walter $P$, Ron $D$. The unfolded protein response: from stress pathway to homeostatic regulation. Science. 2011; 334: 1081-6.

15. Qiao Q, Sun C, Han C, Han N, Zhang M, Li G. Endoplasmic reticulum stress pathway PERK-eIF2alpha confers radioresistance in oropharyngeal carcinoma by activating NF-kappaB. Cancer science. 2017; 108: 1421-31.

16. Li JZ, Lee B, Lee AS. Endoplasmic reticulum stress-induced apoptosis Multiple pathways and activation of p53-up-regulated modulator of apoptosis (PUMA) and NOXA by p53. J Biol Chem. 2006; 281: 7260-70.

17. Pihan P, Carreras-Sureda A, Hetz C. BCL-2 family: integrating stress responses at the ER to control cell demise. Cell Death Differ. 2017; 24: 1478-87.

18. Urra H, Dufey E, Lisbona F, Rojas-Rivera D, Hetz C. When ER stress reaches a dead end. Bba-Mol Cell Res. 2013; 1833: 3507-17.

19. Chaurasia M, Gupta S, Das A, Dwarakanath BS, Simonsen A, Sharma K. Radiation induces EIF2AK3/PERK and ERN1/IRE1 mediated pro-survival autophagy. Autophagy. 2019; 15: 1391-406.

20. Kim KM, Yu TK, Chu HH, Park HS, Jang KY, Moon WS, et al. Expression of ER stress and autophagy-related molecules in human non-small cell lung cancer and premalignant lesions. International journal of cancer Journal international du cancer. 2012; 131: E362-70.

21. Li L, Liu X, Zhou L, Wang W, Liu Z, Cheng Y, et al. Autophagy Plays a Critical Role in Insulin Resistance- Mediated Chemoresistance in Hepatocellular Carcinoma Cells by Regulating the ER Stress. Journal of Cancer. 2018; 9: 4314-24.

22. Jin $X, \operatorname{Li} Q, W u$,, i $P$, Matsumoto $Y$, Furusawa $Y$, et al. Radiosensitization by inhibiting survivin in human hepatoma HepG2 cells to high-LET radiation. Journal of radiation research. 2011; 52: 335-41.

23. Lei G, Zhang Y, Koppula P, Liu X, Zhang J, Lin SH, et al. The role of ferroptosis in ionizing radiation-induced cell death and tumor suppression. Cell research. 2020; 30: 146-62.

24. Jiang P, Mizushima N. LC3- and p62-based biochemical methods for the analysis of autophagy progression in mammalian cells. Methods. 2015; 75: 13-8.

25. Hoyer-Hansen M, Bastholm L, Szyniarowski P, Campanella M, Szabadkai G, Farkas T, et al. Control of macroautophagy by calcium, calmodulin-dependent kinase kinase-beta, and Bcl-2. Molecular cell. 2007; 25: 193-205.

26. Urra H, Dufey E, Avril T, Chevet E, Hetz C. Endoplasmic Reticulum Stress and the Hallmarks of Cancer. Trends Cancer. 2016; 2: 252-62.

27. McGrath EP, Logue SE, Mnich K, Deegan S, Jager R, Gorman AM, et al. The Unfolded Protein Response in Breast Cancer. Cancers (Basel). 2018; 10: 344-65.

28. Nagelkerke A, Bussink J, van der Kogel AJ, Sweep FC, Span PN. The PERK/ATF4/LAMP3-arm of the unfolded protein response affects radioresistance by interfering with the DNA damage response. Radiotherapy and oncology : journal of the European Society for Therapeutic Radiology and Oncology. 2013; 108: 415-21.

29. Dadey D, Kapoor V, Khudanyan A, Thotala D, Hallahan D. Radiation-induced ER stress contributes to survival in glioblastoma cell lines. Cancer Res. 2015; 75: Supplement 15, Meeting Abstract 3323.

30. Verfaillie T, Garg AD, Agostinis P. Targeting ER stress induced apoptosis and inflammation in cancer. Cancer Letters. 2013; 332: 249-64.

31. Torii S, Honda S, Murohashi M, Yamaguchi H, Shimizu S. Autophagy involvement in oncogenesis. Cancer science. 2020.

32. McDonnell SJ, Spiller DG, White MRH, Prior IA, Paraoan L. ER stress-linked autophagy stabilizes apoptosis effector PERP and triggers its co-localization with SERCA2b at ER-plasma membrane junctions. Cell death discovery. 2019; 5: 132

33. Dixon SJ, Lemberg KM, Lamprecht MR, Skouta R, Zaitsev EM, Gleason $\mathrm{CE}$, et al. Ferroptosis: an iron-dependent form of nonapoptotic cell death. Cell. 2012; 149: 1060-72

34. Hassannia B, Vandenabeele P, Vanden Berghe T. Targeting Ferroptosis to Iron Out Cancer. Cancer cell. 2019; 35: 830-49.

35. Su Y, Zhao B, Zhou L, Zhang Z, Shen Y, Lv H, et al. Ferroptosis, a novel pharmacological mechanism of anti-cancer drugs. Cancer Lett. 2020; 483: 127-36.

36. Subburayan K, Thayyullathil F, Pallichankandy S, Cheratta AR, Galadari S. Superoxide-mediated ferroptosis in human cancer cells induced by sodium selenite. Transl Oncol. 2020; 13: 100843.

37. Jiang L, Kon N, Li T, Wang SJ, Su T, Hibshoosh H, et al. Ferroptosis as a p53-mediated activity during tumour suppression. Nature. 2015; 520: 57-62.

38. Kang R, Kroemer G, Tang D. The tumor suppressor protein p53 and the ferroptosis network. Free radical biology \& medicine. 2019; 133: 162-8. 
39. Tang D, Kroemer G. Ferroptosis. Current biology : CB. 2020; 30: R1292-R7.

40. Chen X, Kang R, Kroemer G, Tang D. Broadening horizons: the role of ferroptosis in cancer. Nat Rev Clin Oncol. 2021; 18: 280-96.

41. Tang DL, Kroemer G. Ferroptosis. Curr Biol. 2020; 30: R1292-R7.

42. Gao MH, Monian P, Pan QH, Zhang W, Xiang J, Jiang XJ. Ferroptosis is an autophagic cell death process. Cell Res. 2016; 26: 1021-32.

43. Fernandez-Aroca DM, Roche $O$, Sabater $S$, Pascual-Serra $R$, Ortega-Muelas M, Perez IS, et al. P53 pathway is a major determinant in the radiosensitizing effect of Palbociclib: Implication in cancer therapy. Cancer Letters. 2019; 451: 23-33.

44. Dixon SJ, Patel DN, Welsch M, Skouta R, Lee ED, Hayano M, et al. Pharmacological inhibition of cystine-glutamate exchange induces endoplasmic reticulum stress and ferroptosis. eLife. 2014; 3: e02523.

45. Xie Y, Hou W, Song X, Yu Y, Huang J, Sun X, et al. Ferroptosis: process and function. Cell death and differentiation. 2016; 23: 369-79. 